\title{
PNAS Plus Significance Statements
}

Continuum limit of the vibrational properties of amorphous solids

Hideyuki Mizuno, Hayato Shiba, and Atsushi Ikeda

The thermal properties of crystalline solids follow universal laws that are explained by theories based on phonons. Amorphous solids are also characterized by universal laws that are, however, anomalous with respect to their crystalline counterparts. These anomalies begin to emerge at very low temperatures, suggesting that the vibrational properties of amorphous solids differ from phonons, even in the continuum limit. In this work, we reveal that phonons coexist with soft localized modes in the continuum limit of amorphous solids. Importantly, we discover that the phonons follow the Debye law, whereas the soft localized modes follow another universal nonDebye law. Our findings provide a firm theoretical basis for explaining the thermal anomalies of amorphous solids. (See pp. E9767-E9774.)

Interplay of hemilability and redox activity in models of hydrogenase active sites

Shengda Ding, Pokhraj Ghosh, Marcetta Y. Darensbourg, and Michael B. Hall

Segmentation of the bimetallic electrocatalysts under investigation into a metallodithiolate, bidentate S-donor ligand, and a receiver metal is effective for understanding the proton and electron uptake in $\mathrm{H}_{2}$ evolution reactions. Coexisting actor/reaction-involved ligands, i.e., electron-buffering NO and hemilabile, chelating metallodithiolate, subtly cooperate to control electrocatalytic $\mathrm{H}_{2}$-production mechanisms. Two mechanisms emerge in a single catalyst to yield $\mathrm{H}_{2}$ : protonation of a hydride or reductive elimination from a metal dihydride. A Lewis acid-base pair appears by cleaving the hemilabile thiolate from the metal and serves as the reactive centers to process electrons and protons; a protonation or a reduction on the Lewis pair modulates their electron densities and protects these reactive centers from converting back to a dative bond. (See pp. E9775-E9782.)

Combining disparate data sources for improved poverty prediction and mapping

Neeti Pokhriyal and Damien Christophe Jacques

Spatially finest poverty maps are essential for improved diagnosis and policy planning, especially keeping in view the Sustainable Development Goals. "Big Data" sources like call data records and satellite imagery have shown promise in providing intercensal statistics. This study outlines a computational framework to efficiently combine disparate data sources, like environmental data, and mobile data, to provide more accurate predictions of poverty and its individual dimensions for finest spatial microregions in Senegal. These are validated using the concurrent census data. (See pp. E9783-E9792.)

\section{Enhanced antibacterial activity through the controlled alignment of graphene oxide nanosheets}

Xinglin Lu, Xunda Feng, Jay R. Werber, Chiheng Chu, Ines Zucker, Jae-Hong Kim, Chinedum O. Osuji, and Menachem Elimelech

In biomedical and environmental applications, as well as manufacture and disposal, the interaction of graphene-based nanomaterials (GBNs) with living cells is inevitable and sometimes crucial. While the cytotoxic properties of GBNs are well established, the mechanisms behind the cytotoxicity remain controversial. In this study, we first utilize a magnetic field to form films with aligned graphene oxide (GO), showing that the alignment of sharp $\mathrm{GO}$ edges plays a crucial role in the antibacterial activity. We then demonstrate using model systems that $\mathrm{GO}$ unequivocally induces physical disruption of lipid bilayers and that oxidation stems from a direct electron transfer mechanism. Altogether, our results elucidate the physicochemical, edge-based cytotoxicity of GBNs, while providing guidance for the design of engineered surfaces using GBNs. (See pp. E9793-E9801.)

Reverse chemical ecology: Olfactory proteins from the giant panda and their interactions with putative pheromones and bamboo volatiles

Jiao Zhu, Simona Arena, Silvia Spinelli, Dingzhen Liu, Guiquan Zhang, Rongping Wei, Christian Cambillau, Andrea Scaloni, Guirong Wang, and Paolo Pelosi

The giant panda, an endangered species and a popular emblem, still conceals puzzling unexplored aspects. It shares with bears, to which it is evolutionary related, a carnivorous digestive system but follows a strictly herbivorous diet. The low energy obtained from such poor food accounts for its slow movements and probably, a reduced reproductive activity. Feeding and mating are regulated by olfaction, still poorly investigated in this species at the molecular level. Here, we describe two odorant-binding proteins with complementary affinities to different chemical classes and present the 3D structure of one of them. In a reverse chemical ecology approach, 
which could be adopted for other vertebrates, we use ligandbinding data to suggest putative structures of still unknown sex pheromones. (See pp. E9802-E9810.)

Molecular basis of the 14-3-3 protein-dependent activation of yeast neutral trehalase Nth1

Miroslava Alblova, Aneta Smidova, Vojtech Docekal, Jan Vesely, Petr Herman, Veronika Obsilova, and Tomas Obsil

14-3-3 proteins are conserved scaffolding proteins expressed in all eukaryotic cells, where they regulate the function of several hundreds of partner proteins by constraining their conformation. Yeast neutral trehalases (Nth), enzymes responsible for the hydrolysis of trehalose, compared with trehalases from other organisms, possess distinct structure and regulation involving phosphorylation followed by binding to 14-3-3. Here we present the crystal structures of yeast Nth1 and its complex with the 14-33 protein and propose a molecular mechanism in which 14-33 activates Nth1 by stabilizing the flexible part of its active site. Comparison of the 14-3-3:Nth1 complex structure with those of other 14-3-3 complexes suggests the highly conserved nature of 14-3-3 affects the structures of many client proteins. (See pp. E9811-E9820.)

Role of the nucleotidyl cyclase helical domain in catalytically active dimer formation

Irene Vercellino, Lenka Rezabkova, Vincent Olieric, Yevhen Polyhach, Tobias Weinert, Richard A. Kammerer, Gunnar Jeschke, and Volodymyr M. Korkhov

Adenylyl and guanylyl cyclases are at the core of cellular signaling. Although the molecular mechanisms of the reactions catalyzed by these enzymes are well established, their structures and biophysical properties remain only partially characterized. Here, we report the structure of the cytosolic domain of a mycobacterial adenylyl cyclase Cya, an evolutionary ancestor of mammalian membrane adenylyl cyclases. The structure reveals the helical domain, a highly conserved structural element that links the catalytic and transmembrane portions of Cya. We show how helical domains bring together the catalytic domains to form functionally active dimers. Our data suggest that the diseaselinked mutations in human nucleotidyl cyclases may disrupt the correct assembly of the helical domain, preventing the formation of an active dimeric enzyme. (See pp. E9821-E9828.)

\section{Protocadherin cis-dimer architecture and recognition unit diversity}

Kerry M. Goodman, Rotem Rubinstein, Hanbin Dan, Fabiana Bahna, Seetha Mannepalli, Göran Ahlsén, Chan Aye Thu, Rosemary V. Sampogna, Tom Maniatis, Barry Honig, and Lawrence Shapiro

Pcdhs are cell surface homophilic recognition proteins expressed stochastically to assign individual identities to each neuron. These individual identities ensure repulsion between neurites from the same cell and ensure that neurites from different cells do not repel. However, it is difficult to understand how only $\sim 60$ Pcdh isoforms can provide sufficient diversity for the billions of neurons in vertebrate nervous systems. Here, we report the crystal structure of a Pcdh cis-dimer through which individual Pcdh isoforms associate to form diverse bivalent recognition units. The structure reveals asymmetry in the cis-dimer interaction and suggests restrictions on isoform combinations compatible with cis-dimerization. These findings provide a framework to understand Pcdh cis-dimerization and the compositions of functional repertoires of Pcdh recognition units. (See pp. E9829-E9837.)

Parsing the roles of neck-linker docking and tethered head diffusion in the stepping dynamics of kinesin

Zhechun Zhang, Yonathan Goldtzvik, and D. Thirumalai

Like all motors, the stepping of the two-headed conventional kinesin on the microtubule (MT) is facilitated by conformational changes in the motor domain upon ATP binding and hydrolysis. Numerous experiments have revealed that docking of the 13residue neck linker (NL) to the motor domain of the leading head plays a critical role in propelling the trailing head toward the plus end of the microtubule by nearly $16 \mathrm{~nm}$ in a single step. Surprisingly, our molecular simulations reveal that nearly threequarters of the step occur by stochastic diffusion of the trailing head. Docking of the NL restricts the extent of diffusion, thus forcing the motor to walk with overwhelming probability on a single protofilament of the MT. (See pp. E9838-E9845.)

Probing the cooperativity of Thermoplasma acidophilum proteasome core particle gating by NMR spectroscopy

Rui Huang, Felipe Pérez, and Lewis E. Kay

In the cell, protein degradation occurs, to a large extent, by a barrel-like structure called the proteasome. Degradation is highly regulated, ensuring that only proteins targeted for elimination are removed. This is accomplished through gates that close the proteasome entrance pores, localized to the top and bottom of the structure, as well as through the binding of regulator molecules. Here, we study the properties of the individual gates using solution NMR spectroscopy and a labeling procedure in which a series of molecules are prepared with subunits that have different types of gates. Our results show that gates are formed via residues that have a very high intrinsic propensity to enter the lumen of the proteasome, with each gate behaving independently. (See pp. E9846-E9854.)

\section{Binding kinetics and substrate selectivity in HIV-1 protease-Gag interactions probed at atomic resolution by chemical exchange NMR}

Lalit Deshmukh, Vitali Tugarinov, John M. Louis, and G. Marius Clore

The formation of infectious HIV-1 virions is triggered by sequential proteolysis of the group-specific antigen (Gag) polyprotein by the viral protease. Using chemical exchange-based NMR spectroscopy, we explore, in the context of Gag polyprotein, the formation of productive complexes between protease and Gag cleavage sites located within the intrinsically disordered linkers that connect the globular domains of Gag. We show that the ability of protease to sequentially cleave distinct Gag cleavage sites, which display little to no sequence identity, primarily originates from conformational dynamics of the protease flaps that cover the active site. The protease flaps are actively involved in substrate recognition and regulate the lifetime of productive complexes, allowing protease to differentiate between cognate and noncognate sequences. (See pp. E9855-E9862.) 
Sequences flanking the transmembrane segments facilitate mitochondrial localization and membrane fusion by mitofusin

Xiaofang Huang, Xin Zhou, Xiaoyu Hu, Amit S. Joshi, Xiangyang Guo, Yushan Zhu, Quan Chen, William A. Prinz, and Junjie Hu

Mitochondria constantly connect through membrane fusion. The merging of the outer mitochondrial membrane requires mitofusin (MFN) proteins. MFN is a membrane-anchored GTPase, but whether it is sufficient to achieve fusion, and if so how, is largely unknown. We have taken advantage of a similar GTPase named atlastin (ATL), which mediates fusion of the endoplasmic reticulum (ER), as its mechanism is better understood. Domain swapping experiments show that MFN is capable of fusing membranes, even on the ER. The C-terminal tail of MFN contains an amphipathic helix that promotes fusion. MFN is properly inserted into the mitochondrial membrane with the help of the helix and neighboring hydrophobic residues. These findings provide insight into how mitochondria fuse. (See pp. E9863-E9872.)

Intercellular mRNA trafficking via membrane nanotube-like extensions in mammalian cells

Gal Haimovich, Christopher M. Ecker, Margaret C. Dunagin, Elliott Eggan, Arjun Raj, Jeffrey E. Gerst, and Robert H. Singer

mRNA molecules convey genetic information within cells, beginning from genes in the nucleus to ribosomes in the cell body, where they are translated into proteins. Here we show a mode of transferring genetic information from one cell to another. Contrary to previous publications suggesting that mRNAs transfer via extracellular vesicles, we provide visual and quantitative data showing that mRNAs transfer via membrane nanotubes and direct cell-to-cell contact. We predict that this process has a major role in regulating local cellular environments with respect to tissue development and maintenance and cellular responses to stress, interactions with parasites, tissue transplants, and the tumor microenvironment. (See pp. E9873-E9882.)

Probing and manipulating intracellular membrane traffic by microinjection of artificial vesicles

Seiichi Koike and Reinhard Jahn

In eukaryotic cells, membranous organelles exchange trafficking vesicles that bud from the precursor and are transported to the target membrane where they fuse. Many of the required proteins are known. Moreover, some steps such as budding and fusion have been reconstituted using artificial membranes and a minimalistic set of proteins. However, it is not known whether these vesicles are functional in the complex environment of a cell. Here, we have introduced such artificial vesicles into living cells and find that only few components are needed for function in membrane traffic, with all other factors recruited from the cytoplasm. Thus, eukaryotic cells have a remarkable capacity for selforganization that can be exploited for targeting tags to specific regions in the cell. (See pp. E9883-E9892.)

\section{Patterns of conserved gp120 epitope presentation on} attached HIV-1 virions

Meron Mengistu, Ai-hui Tang, James S. Foulke Jr., Thomas A. Blanpied, Mileidy W. Gonzalez, John L. Spouge, Robert C. Gallo, George K. Lewis, and Anthony L. DeVico

A complete picture of HIV antigenicity during early replication is needed to elucidate the full range of options for controlling infection through humoral immunity. The HIV envelope protein, gp120, experiences key structural rearrangements during host cell attachment, leading to exposure of highly conserved epitopes on the virion surface. These epitopes enable Fc-mediated antiviral effector functions that may be relevant to HIV prevention. Here, we used 3D superresolution microscopy to show how gp120 epitopes are rapidly exposed distal to cell-virus interfaces, introducing the opportunity for unconstrained antibody binding. These previously unrecognized facets of HIV antigenicity further define relationships between retroviral infection and immunity and should facilitate the development of antibody-based approaches for HIV prevention. (See pp. E9893-E9902.)

\section{A vimentin binding small molecule leads to mitotic} disruption in mesenchymal cancers

Michael J. Bollong, Mika Pietilä, Aaron D. Pearson, Tapasree Roy Sarkar, Insha Ahmad, Rama Soundararajan, Costas A. Lyssiotis, Sendurai A. Mani, Peter G. Schultz, and Luke L. Lairson

Cancer cells derived from mesenchymal tissues or induced to adopt a mesenchymal state have been demonstrated to be largely resistant to standard chemotherapies, necessitating the identification of new effective treatment strategies. From a high throughput screen, we have discovered FiVe1, a compound capable of irreversibly inhibiting the growth of mesenchymally transformed cancer cells by binding to and interfering with the organization and phosphorylation of vimentin (VIM) during mitosis. In contrast to the many naturally and synthetically derived compounds targeting microtubules, we report that chemically targeting an intermediate filament protein, VIM, promotes mitotic catastrophe. As VIM expression is restricted to mesenchymal cells, these results provide a mechanistic basis toward developing genotype-selective chemotherapeutics for the treatment of mesenchymal cancers. (See pp. E9903-E9912.)

Relevance of iPSC-derived human PGC-like cells at the surface of embryoid bodies to prechemotaxis migrating PGCs

Shino Mitsunaga, Junko Odajima, Shiomi Yawata, Keiko Shioda, Chie Owa, Kurt J. Isselbacher, Jacob H. Hanna, and Toshi Shioda

Human primordial germ cell-like cells (hPGCLCs) generated from pluripotent stem cells in vitro hold promise, with broad applications for studies of human germline cells. We show that hPGCLCs generated using several distinct protocols are transcriptomally comparable and that primed pluripotency human iPSCs gain competence to generate hPGCLCs after only 72 hours of reprogramming toward ERK-independent state-naïve pluripotency. hPGCLCs were localized in the outermost surface layer of embryoid bodies and strongly expressed CXCR4. Live cell imaging showed active migratory activity of hPGCLCs, and their exposure to the CXCR4 ligand CXCL12/SDF-1 induced enriched expression of promigratory genes and antiapoptotic genes. These results support the resemblance of hPGCLCs to prechemotaxis human embryonic primordial germ cells migrating in the midline region of embryos. (See pp. E9913-E9922.)

\section{Contaminants of emerging concern affect Trichoplusia} ni growth and development on artificial diets and a key host plant

Marcus J. Pennington, Jason A. Rothman, Stacia L. Dudley, Michael B. Jones, Quinn S. McFrederick, Jay Gan, and John T. Trumble

Rising temperatures, drought, and growing human populations are increasing demand for reclaimed wastewater for agricultural use. 
However, wastewater often contains biologically active, pseudopersistent pharmaceuticals, even after passage through a water treatment facility. We determined that the biology, life histories, and microbial communities of an agricultural pest insect were altered when reared on artificial diets or plants irrigated by these chemicals. In this study, pharmaceuticals translocated through plants have been shown to negatively affect the biology of an agriculturally important insect. The responses to these pharmaceuticals could have implications for integrating pest management practices and for understanding the effects of reclaimed water on agricultural ecosystems that are critical for the sustainability of our food supply. (See pp. E9923-E9931.)

\section{Seasonally fluctuating selection can maintain polymorphism at many loci via segregation lift}

Meike J. Wittmann, Alan O. Bergland, Marcus W. Feldman, Paul S. Schmidt, and Dmitri A. Petrov

A key question in evolutionary biology is: What maintains the abundant genetic variation observed in natural populations? Many organisms experience some seasonality in their habitats, and, if they have multiple generations per year, seasonally fluctuating selection is a potentially powerful mechanism to maintain polymorphism. However, previous research has argued that this occurs rarely. Inspired by recent empirical findings, we reevaluate the potential of seasonally fluctuating selection to simultaneously maintain polymorphism at many loci in the genome. We obtain a more general condition for the maintenance of multilocus polymorphism by seasonally fluctuating selection. This condition may plausibly be satisfied for many species and does not suffer from problems of previous models. (See pp. E9932-E9941.)

The immunopeptidomic landscape of ovarian carcinomas Heiko Schuster, Janet K. Peper, Hans-Christian Bösmüller, Kevin Röhle, Linus Backert, Tatjana Bilich, Britta Ney, Markus W. Löffler, Daniel J. Kowalewski, Nico Trautwein, Armin Rabsteyn, Tobias Engler, Sabine Braun, Sebastian P. Haen, Juliane S. Walz, Barbara Schmid-Horch, Sara Y. Brucker, Diethelm Wallwiener, Oliver Kohlbacher, Falko Fend, Hans-Georg Rammensee, Stefan Stevanović, Annette Staebler, and Philipp Wagner

Despite the revolution in cancer therapy initiated by checkpoint inhibitors, durable clinical responses remain sporadic in many types of cancer, including ovarian cancer. Understanding which antigens are essentially presented by tumor cells and further able to be recognized by $T$ cells provides a major step toward novel effective targeted immunotherapies. In this study, we comprehensively analyzed the immunopeptidomic landscape of ovarian carcinoma and compared it to variety of benign sources to identify antigens exclusively presented on tumor cells. With personalized therapies moving into the focus of clinical cancer therapy, we further present insights on how gene-expression analysis and immunohistochemistry can support antigen selection for individualized immunotherapy. (See pp. E9942-E9951.)

\section{Visual experience sculpts whole-cortex spontaneous infraslow activity patterns through an Arc-dependent mechanism}

Andrew W. Kraft, Anish Mitra, Adam Q. Bauer, Abraham Z. Snyder, Marcus E. Raichle, Joseph P. Culver, and Jin-Moo Lee

Visual critical periods are essential for shaping the normal mature responsiveness of visual cortex neurons; however, it is unclear whether early visual experience influences functional network organization. Here we demonstrate that monocular and binocular visual deprivation (MD and $\mathrm{BD}$ ) have distinct effects on cortical functional connectivity (FC). MD induces $F C$ changes restricted to the visual system, while BD results in widespread FC changes between disparate brain regions. These results suggest that early exposure to altered vision (MD) induces intranetwork change, but absent vision (BD) drives internetwork change, reminiscent of cross-modal plasticity. Moreover, this early experience-driven FC change was attenuated by deletion of Arc, a gene critical for synaptic plasticity, suggesting that network relationships are modulated by changes in synaptic strengths. (See pp. E9952-E9961.)

\section{Electrical synapses mediate synergism between pheromone and food odors in Drosophila melanogaster}

Sudeshna Das, Federica Trona, Mohammed A. Khallaf, Elisa Schuh, Markus Knaden, Bill S. Hansson, and Silke Sachse

We elucidated the neuronal mechanism underlying the interaction of two ecologically relevant odors in the fly brain. Our study demonstrates that exposure to the male-produced sex pheromone cis-vaccenyl acetate, in combination with the complex food odor vinegar, evokes an enhanced and synergistic functional response in the primary olfactory center of virgin female flies. This effect arises within the neuronal network of the antennal lobe and is mediated by electrical synapses. The synergistic response in virgin females leads to an increased sensitivity to the pheromone, and therefore an enhanced female receptivity during courtship. This mechanism is highly useful, since it promotes mating in females when food is present: that is, when the nutritional supply of the female and its offspring is guaranteed. (See pp. E9962-E9971.)

\section{Silent memory engrams as the basis for retrograde amnesia}

Dheeraj S. Roy, Shruti Muralidhar, Lillian M. Smith, and Susumu Tonegawa

We previously discovered that memory is retrieved robustly from protein synthesis inhibitor-induced retrograde amnesia by optogenetic activation of engram cells. Connectivity of engram cells correlates with memory information storage under amnesia, even though these amnesic engram cells lack learning-induced augmented synaptic strength. We term this state of engrams as "silent engrams." The significance of this study is threefold: first, the silent state of the engram can last for a prolonged period (at least $8 \mathrm{~d}$ post encoding); second, connectivity between engram cell ensembles is causally linked to optogenetic recall and hence memory information storage; and third, there is a molecular genetic method to convert an engram from a silent state to an active state. (See pp. E9972-E9979.)

\section{Active site voltage clamp fluorometry of the sodium glucose cotransporter hSGLT1}

Edurne Gorraitz, Bruce A. Hirayama, Aviv Paz, Ernest M. Wright, and Donald D. F. Loo

Site-directed fluorometry was used to understand conformational changes of the $\mathrm{Na}^{+} /$glucose symporter. SGLT1 functions by a mechanism where the substrate-binding site alternates between the two faces of the membrane, but little is known about the underlying conformational changes. Rhodamines were covalently inserted into the substrate cavity, and changes of fluorescence were measured in real time with the opening and closing of the outer gate as SGLT1 was driven between inward and outward conformations using voltage jumps. Structural modeling indicated that the quenching with gating opening was due to an increased solvation of rhodamine and an increase in polar 
residues lining the wall of the cavity. This experimental approach will lead to a better understanding of the mechanism of membrane transport. (See pp. E9980-E9988.)

Transcriptomes of major renal collecting duct cell types in mouse identified by single-cell RNA-seq

Lihe Chen, Jae Wook Lee, Chung-Lin Chou, Anil V. Nair, Maria A. Battistone, Teodor G. Păunescu, Maria Merkulova, Sylvie Breton, Jill W. Verlander, Susan M. Wall, Dennis Brown, Maurice B. Burg, and Mark A. Knepper

A long-term goal in mammalian biology is to identify the genes expressed in every cell type of the body. In the kidney, the expressed genes (i.e., transcriptome) of all epithelial cell types have already been identified with the exception of the cells that make up the renal collecting duct, which is responsible for regulation of blood pressure and body fluid composition. Here, singlecell RNA-sequencing was used in mouse to identify transcriptomes for the major collecting duct cell types: type A intercalated cells, type B intercalated cells, and principal cells. The information was used to create a publicly accessible online resource. The data allowed identification of genes that are selectively expressed in each cell type, which is informative for cell-level understanding of physiology and pathophysiology. (See pp. E9989-E9998.)

Genome diversity of tuber-bearing Solanum uncovers complex evolutionary history and targets of domestication in the cultivated potato

Michael A. Hardigan, F. Parker E. Laimbeer, Linsey Newton, Emily Crisovan, John P. Hamilton, Brieanne Vaillancourt, Krystle Wiegert-Rininger, Joshua C. Wood, David S. Douches, Eva M. Farré, Richard E. Veilleux, and C. Robin Buell

Worldwide, potato is the third most important crop grown for direct human consumption, but breeders have struggled to produce new varieties that outperform those released over a century ago, as evidenced by the most widely grown North American cultivar (Russet Burbank) released in 1876. Despite its importance, potato genetic diversity at the whole-genome level remains largely unexplored. Analysis of cultivated potato and its wild relatives using modern genomics approaches can provide insight into the genomic diversity of extant germplasm, reveal historic introgressions and hybridization events, and identify genes targeted during domestication that control variance for agricultural traits, all critical information to address food security in 21 st century agriculture. (See pp. E9999-E10008.)

MPSR1 is a cytoplasmic PQC E3 ligase for eliminating emergent misfolded proteins in Arabidopsis thaliana

Jong Hum Kim, Seok Keun Cho, Tae Rin Oh, Moon Young Ryu, Seong Wook Yang, and Woo Taek Kim

The essential roles of cytoplasmic E3 ligases in the protein quality control (PQC) pathways have been increasingly highlighted in yeast and animal studies. However, in plants, only CHIP E3 ligase has been characterized, while the knowledge of cytoplasmic PQC E3 ligases remains rudimentary. Misfolded Protein Sensing RING E3 ligase 1 (MPSR1), a self-regulatory sensor system that functions only in the occurrence of misfolded proteins, is an identified cytoplasmic PQC E3 ligase in plants that directly recognizes emergent misfolded proteins independently of chaperones. In addition, MPSR1 sustains the integrity and activity of the 26S proteasome under proteotoxic stress. Given that MPSR1 RING E3 ligase is well conserved in eukaryotes, this study sheds light on a $\mathrm{PQC}$ pathway that is present particularly in plants and beyond. (See pp. E10009-E10017.)

Global analysis of ribosome-associated noncoding RNAs unveils new modes of translational regulation

Jérémie Bazin, Katja Baerenfaller, Sager J. Gosai, Brian D. Gregory, Martin Crespi, and Julia Bailey-Serres

Noncoding RNAs are an underexplored reservoir of regulatory molecules in eukaryotes. We analyzed the environmental response of roots to phosphorus $(\mathrm{Pi})$ nutrition to understand how a change in availability of an essential element is managed. Pi availability influenced translational regulation mediated by small upstream ORFs on protein-coding mRNAs. Discovery, classification, and evaluation of long noncoding RNAs (IncRNAs) associated with translating ribosomes uncovered diverse new examples of translational regulation. These included Pi-regulated small peptide synthesis, ribosome-coupled phased small interfering RNA production, and the translational regulation of natural antisense RNAs and other regulatory RNAs. This study demonstrates that translational control contributes to the stability and activity of regulatory RNAs, providing an avenue for manipulation of traits. (See pp. E10018-E10027.) 